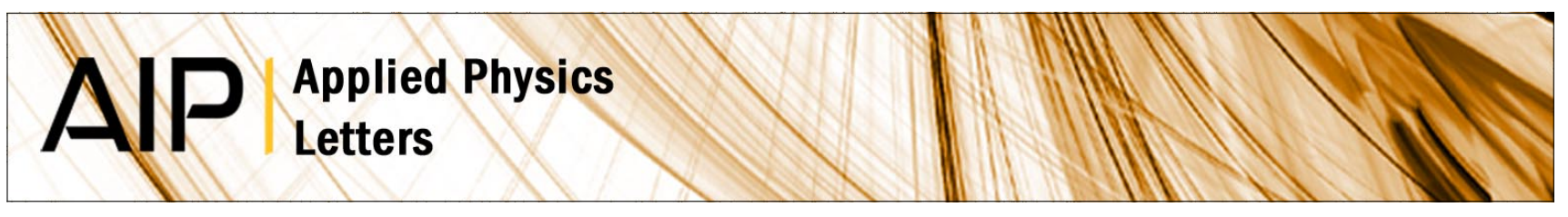

\title{
Defect structures in metallic photonic crystals
}

E. Özbay, B. Temelkuran, M. Sigalas, G. Tuttle, C. M. Soukoulis et al.

Citation: Appl. Phys. Lett. 69, 3797 (1996); doi: 10.1063/1.117002

View online: http://dx.doi.org/10.1063/1.117002

View Table of Contents: http://apl.aip.org/resource/1/APPLAB/v69/i25

Published by the American Institute of Physics.

Additional information on Appl. Phys. Lett.

Journal Homepage: http://apl.aip.org/

Journal Information: http://apl.aip.org/about/about_the_journal

Top downloads: http://apl.aip.org/features/most_downloaded

Information for Authors: http://apl.aip.org/authors

\section{ADVERTISEMENT}

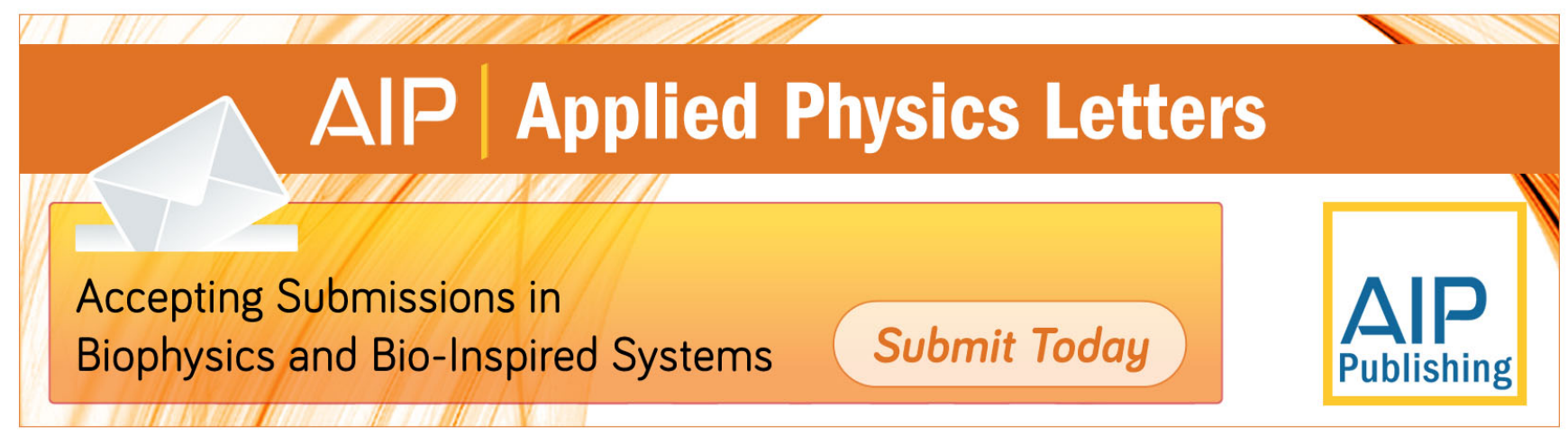




\title{
Defect structures in metallic photonic crystals
}

\author{
E. Özbay ${ }^{a)}$ and B. Temelkuran \\ Department of Physics, Bilkent University, Bilkent, Ankara 06533, Turkey \\ M. Sigalas, G. Tuttle, C. M. Soukoulis, and K. M. Ho \\ Ames Laboratory and Microelectronics Research Center, Iowa State University, Ames, Iowa 50011
}

(Received 28 August 1996; accepted for publication 16 October 1996)

\begin{abstract}
We have investigated metallic photonic crystals built around a layer-by-layer geometry. Two different crystal structures (face-centered-tetragonal and tetragonal) were built and their properties were compared. We obtained rejection rates of 7-8 dB per layer from both metallic crystals. Defect modes created by removing rods resulted in high peak transmission $(80 \%)$, and high quality factors (1740). Our measurements were in good agreement with theoretical simulations. (C) 1996
\end{abstract} American Institute of Physics. [S0003-6951(96)04251-9]

Photonic crystals are three-dimensional periodic dielectric structures where the propagation of electromagnetic (EM) waves can be forbidden for a certain range of frequencies. ${ }^{1-3}$ Early attempts to use these structures in applications like thresholdless semiconductor lasers ${ }^{4}$ and single-mode light-emitting diodes ${ }^{5,6}$ have suffered from the difficulties associated with fabricating submicron features needed to achieve a band gap at optical frequencies. On the other hand, fabricating photonic band gap (PBG) structures at microwave and millimeter-wave scales have been more successful, ${ }^{7}$ with numerous demonstrations of PBG-based applications like high directivity millimeter wave antennas, ${ }^{8,9}$ high-quality resonators, ${ }^{10}$ microwave cavities for accelerators, ${ }^{11}$ and efficient microwave reflectors. ${ }^{12}$

Although the employment of photonic crystals made of dielectric materials have been successful in various applications, some of their properties restrict the wide usage of these materials. First, the rejection from the dielectric based photonic crystals are typically limited to a maximum of $3-4 \mathrm{~dB}$ per layer. ${ }^{13}$ This means that an application requiring $40 \mathrm{~dB}$ isolation would need at least 10 layers, which is often too large to meet space constraints. Furthermore, for applications around $1-10 \mathrm{GHz}$ range, the relatively large surface area of a photonic crystal becomes another limiting factor. As an example, a dielectric-based dielectric photonic crystal with a band gap centered at $2 \mathrm{GHz}$ will have a surface area larger than one square meter which is again not practical for most applications. As has been suggested by other researchers, these problems can be solved by introducing metals to photonic crystals. ${ }^{14-16}$ Although the metals exhibit high absorption at optical frequencies, they act like nearly perfect conductors at lower microwave and millimeter-wave frequencies which minimizes the problems related to absorption.

In our investigations of metallic photonic crystals, we first examined the face-centered-tetragonal (fct) structure shown in Fig. 1(a). The stacking sequence repeats every four layers, corresponding to a single unit cell in the stacking direction. ${ }^{17}$ Previously, we used this structure to make dielectric-based photonic crystals with a full band gap at frequencies ranging from microwave ${ }^{18}$ to far infrared. ${ }^{19}$ In addition to the fct structure, we also used the tetragonal struc-

${ }^{a)}$ Electronic mail: ozbay@fen.bilkent.edu.tr ture in our examination of metallic photonic crystals. This structure which is depicted in Fig. 1(b), has a two-layer unit cell in the stacking direction. The metallic rods used in this study were $2.8 \mathrm{~mm}$ wide, $2.5 \mathrm{~mm}$ thick, and $120 \mathrm{~mm}$ long. The center to center distance between adjacent parallel rods was $7.6 \mathrm{~mm}$. The rods were obtained by machining 150 $\times 150 \times 5 \mathrm{~mm}$ aluminum blocks. The blocks were machined from bottom and top surfaces to create parallel rods on each side. Each block contained two layers of metallic rods, where the rods on the top side were perpendicular to the rods on the bottom side. These blocks were then stacked to form either the fct or the tetragonal structures depicted in Fig. 1.

We measured the transmission properties of the metallic structures using a Hewlett-Packard 8510C network analyzer. Standard gain horn antennas were used to transmit and receive the EM radiation. Surroundings of the test setup were covered with absorbers to build an anechoic chamber resulting in a sensitivity of $85 \mathrm{~dB}$. Three separate pairs of standard gain horn antennas were used to cover the $8-26 \mathrm{GHz}$ measurement range.

We first measured the transmission properties of the fct and tetragonal metallic PBG crystals as a function of number of stacked layers. Figures 2(a) and 2(b) compare the transmission properties of 5 different crystals made of 2, 4, 6, 8, and 10 layers of metallic rods. As can be seen from the plots, both structures yielded band gaps with upper edges around

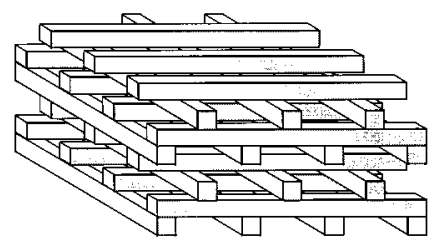

(a)

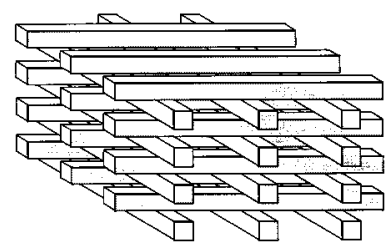

(b) 

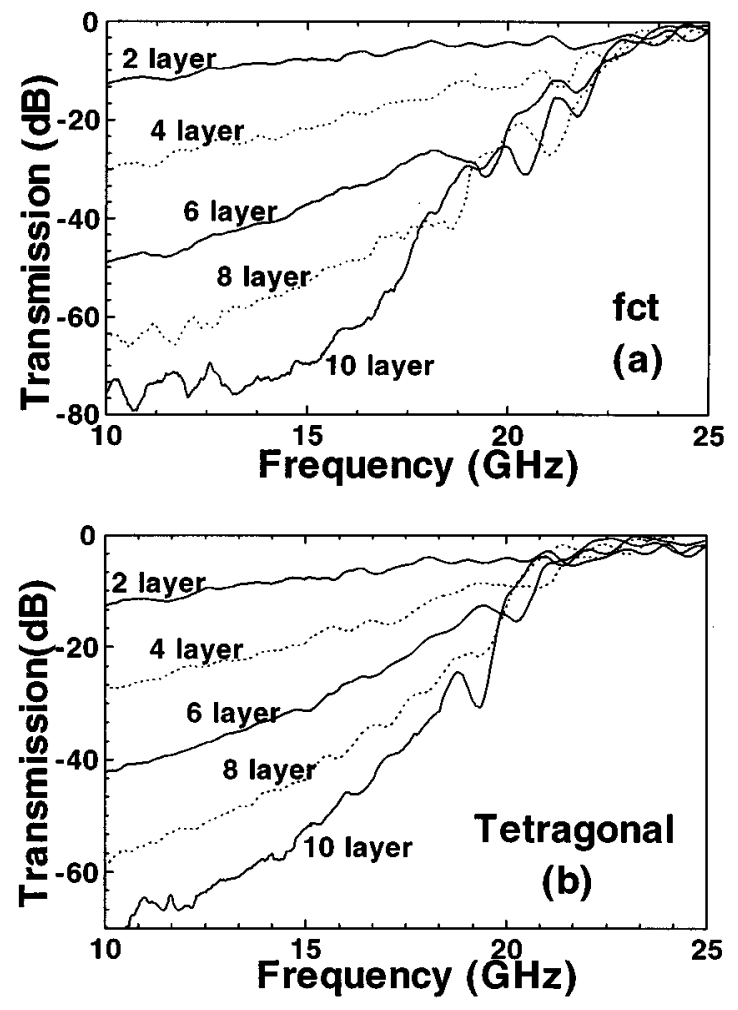

FIG. 2. (a) Transmission properties of (a) fct crystals, and (b) tetragonal crystals for $2,4,6,8$, and 10 layers.

$20 \mathrm{GHz}$ and no lower edge. This large "metallicity gap", extending from zero frequency to a cut-off value that is dependent on the periodicity is typical of metallic PBGs. Within the metallicity gap, the typical rejection factors of our crystals were around 7-8 dB per layer. This was a significant improvement over the dielectric based structures which had a maximum rejection of 3-4 dB per layer. Furthermore, since the metallicity gap extends down to lower frequencies, the metallic structures can also be used at frequencies between 1-10 GHz without any surface area problems.

After observing the EM wave rejection properties of layer-by-layer metallic crystals, we measured defect structures built around these crystals. The defect structures were formed by removing rods from the crystal. Figure 3(a) shows the transmission properties of a 14 layer tetragonal type PBG crystal where 7 th layer is the defect layer with a single missing rod. The defect mode occurred at $17.2 \mathrm{GHz}$, and had a peak transmission amplitude of $-7 \mathrm{~dB}$ with a $Q$ factor (quality factor, defined as the center frequency divided by the peak's full width at half-maximum) of 750. The electric field polarization vector of the incident EM wave $e$ was parallel to the rods of the defect layer and no defect mode was present when $e$ was perpendicular to the rods of the defect layer. The $Q$ factor of the defect mode can be further increased by increasing the number of layers. Figure 3(b) shows the characteristics of an 18 layer structure (where the 9th layer was chosen as the defect layer) on an expanded frequency scale. This defect mode exhibited a peak transmission of $-19 \mathrm{~dB}$, along with a $Q$ factor of 1740 .

The defect structures built around the fct structure gave rather limited performance. A defect structure similar to the one described earlier (14 layers) gave a peak transmission
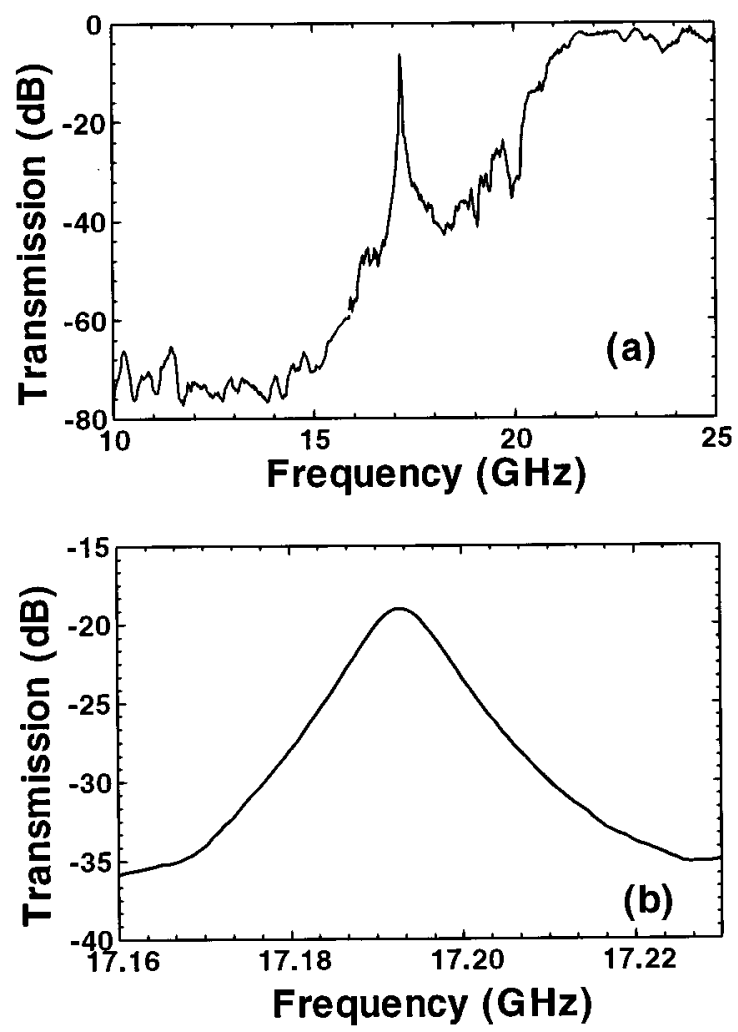

FIG. 3. (a) Transmission characteristics of a tetragonal 14 layer defect. (b) Expanded frequency scale of an 18 layer defect structure.

amplitude of $-30 \mathrm{~dB}$ along with a $Q$ factor of 950 . The peak transmission quickly dropped to below noise level when we increased the number of layers. So, the tetragonal based metallic defect structures were found to be superior to fct based metallic defect structures in terms of peak amplitude and the maximum achievable $Q$ factor.

The transfer-matrix method ${ }^{20,21}$ (TMM) was used to calculate the EM transmission through the metallic structures. Since the TMM method requires periodicity in the directions parallel to the interfaces, we examined the case of a planar defect, made by removing all rods in a single layer. A 10layer thick tetragonal structure where the 5th layer was chosen as the defect layer, was used for both theoretical simulations and experimental measurements. Figure 4 compares

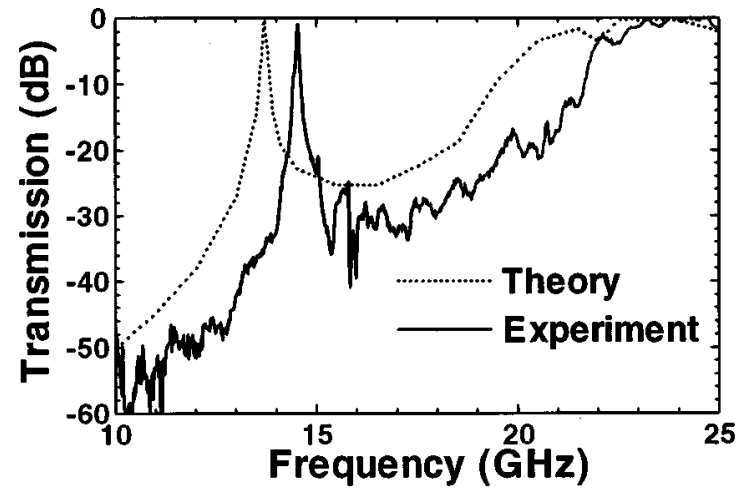

FIG. 4. Comparison of the experimental transmission properties (solid line) of the metallic planar defect structure with the theoretical simulations (dashed line). 
the predicted theoretical transmission with the experimental results. As can be seen from the plot, theory and experiment were in good agreement. A peak transmission $0.9 \mathrm{~dB}$ below the incident signal (corresponding to $80 \%$ transmission) and a $Q$ factor of 400 were measured, while theory predicted a defect mode with $-0.2 \mathrm{~dB}$ peak transmission and a $Q$ factor of 440. The calculated defect frequency $(13.7 \mathrm{GHz})$ was slightly off from the measured defect frequency $(14.5 \mathrm{GHz})$. The discrepancy was due to the limitations coming from simulating the real structure by a discrete computer model. In the present calculations, we divided each unit cell into $16 \times 16 \times 10$ cells. $^{20,21}$ By increasing the number of cells, the model gets closer to the real structure. However, the memory and computer time requirements for a higher number of cells make those calculations almost impossible.

In summary, we have investigated the properties of metallic layer-by-layer photonic crystals. An average rejection rate of $8 \mathrm{~dB}$ per layer was measured. Defect modes created by removing rods resulted in high peak transmission $(80 \%)$, and high quality factors (1740). Our measurements were in good agreement with the theoretical simulations. To our knowledge, our defect measurements correspond to the highest quality factors reported for metallic photonic crystals in scientific literature.

The authors would like to thank R. Biswas for useful discussions. This work is supported by the Turkish Scientific and Technical Research Council of Turkey (TÜBITAK) under Contract No. EEEAG-156, National Science Foundation Grant No. INT-9512812, and NATO-Collaborative Research Grant No. 950079. Ames Laboratory is operated for the U. S. Department of Energy by Iowa State University under Contract No. W-7405-Eng-82.
${ }^{1}$ E. Yablonovitch, T. J. Gmitter, and K. M. Leung, Phys. Rev. Lett. 67, 2295 (1991).

${ }^{2}$ K. M. Ho, C. T. Chan, and C. M. Soukoulis, Phys. Rev. Lett. 65, 3152 (1990).

${ }^{3}$ For a review, see the articles in Photonic Band Gap Materials, edited by C. M. Soukoulis (Plenum, New York, 1996).

${ }^{4}$ P. R. Villenevue, S. Fan, J. D. Joannopoulos, K. Y. Lim, G. S. Petrich, L. A. Kolodjeski, and R. Reif, Appl. Phys. Lett. 67, 167 (1995).

${ }^{5}$ P. L. Gourley, J. R. Wendt, G. A. Vawter, T. M. Brennan, and B. E. Hammons, Appl. Phys. Lett. 6, 687 (1994)

${ }^{6}$ J. P. Dowling, M. Scalora, M. J. Bloemer, and C. M. Bowden, J. Appl. Phys. 75, 1896 (1994).

${ }^{7}$ E. Ozbay, E. Michel, G. Tuttle, M. Sigalas, R. Biswas, and K. M. Ho, Appl. Phys. Lett. 64, 2059 (1994).

${ }^{8}$ S. D. Cheng, R. Biswas, E. Özbay, J. S. McCalmont, G. Tuttle, and K. M. Ho, Appl. Phys. Lett. 67, 3399 (1995).

${ }^{9}$ E. R. Brown and O. B. McMahon, Appl. Phys. Lett. 68, 1300 (1996).

${ }^{10}$ C. J. Maggiore, A. M. Clogston, G. Spalek, W. C. Sailor, and F. M. Mueller, Appl. Phys. Lett. 64, 1451 (1994).

${ }^{11}$ D. R. Smith, S. Shultz, N. Kroll, M. Sigalas, K. M. Ho, and C. M. Soukoulis, Appl. Phys. Lett. 65, 645 (1994).

${ }^{12}$ E. R. Brown and O. B. McMahon, Appl. Phys. Lett. 67, 2138 (1995).

${ }^{13}$ E. Ozbay, J. S. McCalmont, G. Tuttle, R. Biswas, M. M. Sigalas, C. Soukoulis, and K. M. Ho, Appl. Phys. Lett. 67, 1969 (1995).

${ }^{14}$ M. Sigalas, C. T. Chan, K. M. Ho, and C. M. Soukoulis, Phys. Rev. B 52, 11744 (1995)

${ }^{15}$ D. F. Sievenpiper, M. E. Sickmiller, and E. Yablonovitch, Phys. Rev. Lett. 76, 2480 (1996).

${ }^{16}$ J. S. McCalmont, M. Sigalas, G. Tuttle, K. M. Ho, and C. M. Soukoulis, Appl. Phys. Lett. 68, 2759 (1996).

${ }^{17}$ K. M. Ho, C. T. Chan, C. M. Soukoulis, R. Biswas, and M. Sigalas, Solid State Commun. 89, 413 (1994).

${ }^{18}$ E. Ozbay, A. Abeyta, G. Tuttle, M. Tringides, R. Biswas, C. Soukoulis, C. T. Chan, and K. M. Ho, Phys. Rev. B 50, 1945 (1994).

${ }^{19}$ E. Ozbay, E. Michel, G. Tuttle, R. Biswas, K. M. Ho, J. Bostak, and D. M. Bloom, Opt. Lett. 19, 1155 (1994).

${ }^{20}$ J. B. Pendry and A. MacKinnon, Phys. Rev. Lett. 69, 2722 (1992).

${ }^{21}$ M. M. Sigalas, C. M. Soukoulis, E. N. Economou, C. T. Chan, and K. M. Ho, Phys. Rev. B 48, 14121 (1993). 